\title{
VOLUME OF RIEMANNIAN MANIFOLDS, GEOMETRIC INEQUALITIES, AND HOMOTOPY THEORY
}

\author{
MIKHAIL G. KATZ AND ALEXANDER I. SUCIU \\ Dedicated to Mel Rothenberg on his $65^{\text {th }}$ birthday
}

\begin{abstract}
We outline the current state of knowledge regarding geometric inequalities of systolic type, and prove new results, including systolic freedom in dimension 4. Namely, every compact, orientable, smooth 4-manifold $X$ admits metrics of arbitrarily small volume such that every orientable, immersed surface of smaller than unit area is necessarily null-homologous in $X$. In other words, orientable 4-manifolds are 2-systolically free. More generally, let $m$ be a positive even integer, and let $n>m$. Then all manifolds of dimension at most $n$ are $m$-systolically free (modulo torsion) if all $k$-skeleta, $m+1 \leq k \leq n$, of the loop space $\Omega\left(S^{m+1}\right)$ are $m$-systolically free.
\end{abstract}

\section{IntRoduction}

Our purpose here is both to present new results and to outline the current state of knowledge regarding geometric inequalities of systolic type. One of the new results (proved in $\$ \otimes$ ) is the following.

Theorem 1.1. Every compact, orientable, smooth 4-manifold $X$ admits metrics of arbitrarily small volume such that every orientable, immersed surface of smaller than unit area is necessarily null-homologous in $X$.

In other words, orientable 4-manifolds are 2 -systolically free. A precise definition of systolic freedom will be given in $\$$ 3 . Roughly speaking, a $k$-systole of a Riemannian manifold $(X, g)$ is the least volume of a non-trivial $k$-cycle in $(X, g)$. To say that $X$ is $k$-systolically free means that the volume of $g$ imposes no upper bound upon the $k$-systole. One can view this result as a way of producing metrics for which the systole and the mass in the middle dimension do not agree ( $c f$. Theorem 2.3 and 11).

Remark 1.2. Consider sufficiently small perturbations $g$ of the Fubini-Study metric $g_{0}$ on $\mathbb{C P}^{2}$, scaled to the same volume as $g_{0}$. M. Gromov proved in [13] (Corollary, p. 309) that one can always find embedded 2-spheres, homologous to the projective line $\mathbb{C P}^{1} \subset \mathbb{C P}^{2}$, of $g$-area no greater than that of $\mathbb{C P}^{1}$ for the $g_{0}$ metric. On the other hand, our Theorem 1.1 shows that there may be no such spheres if we go far away from the Fubini-Study metric in the space of Riemannian metrics on $\mathbb{C P}^{2}$. We see that here local and global diverge.

1991 Mathematics Subject Classification. Primary 53C23; Secondary 55Q15.

Key words and phrases. volume, systole, stable systole, systolic freedom, coarea inequality, isoperimetric inequality, surgery, Whitehead product, loop space, Eilenberg-MacLane space.

To appear in the Rothenberg Festschrift (M. Farber, W. Lück, S. Weinberger, eds.), Contemporary Math., Amer. Math. Soc., Providence, RI. Available at http://xxx.lanl.gov/abs/ nath.DG/9810172. 
Remark 1.3. The conclusion of the theorem may well be as strong as possible, in the sense that non-orientable surfaces of small area (constrained by the volume of the ambient manifold) may always be present. M. Freedman 10 raised the question of what happens with the systoles defined using homology with $\mathbb{Z}_{2}$-coefficients. Gromov 16] conjectures that the systolic inequalities are true in this case, i.e., our theorem above on systolic freedom has no $\mathbb{Z}_{2}$-analogue. As the example in Remark A.4 illustrates, $\mathbb{Z}_{2}$-cycles may have asymptotically smaller area growth than $\mathbb{Z}$-cycles, supporting the conjecture that volume is a constraint upon the $\mathbb{Z}_{2}$-systoles. The exact boundaries between freedom and constraint are yet to be described.

In the 4-dimensional case, we reduce the $\bmod 2$ problem to $\mathbb{C P}^{2}$ and $S^{2} \times S^{2}$, in the following sense.

Theorem 1.4. All compact, orientable, smooth 4-manifolds are 2-systolically free over $\mathbb{Z}_{2}$ if and only if $S^{2} \times S^{2}$ and $\mathbb{C P}^{2}$ are 2 -systolically free over $\mathbb{Z}_{2}$.

Our Theorem 1.1 permits the following generalization of the results obtained by the authors in collaboration with I. Babenko in [3.

Theorem 1.5. Every compact, smooth manifold of dimension $n=2 m \geq 4$ admits metrics of arbitrarily small $n$-dimensional volume such that every orientable, immersed middle-dimensional submanifold of smaller than unit m-volume represents a torsion class in $m$-dimensional homology with integer coefficients.

In other words, $2 m$-dimensional manifolds are $m$-systolically free (modulo torsion). The term 'systolic freedom (modulo torsion)' is also explained in \$3. For manifolds of dimension larger than $2 m$, we prove the following.

Theorem 1.6. Let $m \geq 2$ be an even integer, and let $n>m$. Then all manifolds of dimension at most $n$ are $m$-systolically free (modulo torsion) if all $k$-skeleta, $m+1 \leq k \leq n$, of the based loop space $\Omega\left(S^{m+1}\right)$ are $m$-systolically free.

The paper is organized as follows. In \$2, we define systoles and give representative proofs of inequalities exhibiting constraint imposed by the volume (of the ambient manifold) on them. In $\$ 3$, we explain the first example of systolic freedom (i.e., the absence of such constraint), due to M. Gromov, and give related definitions and historical comments. In $\S$, we prove Theorem 1.6. In $\S 5$, we introduce a 'carving-up' technique, with the goal of reducing the problem to the case when the middle Betti number is at most 2. In $\S 6$, we prove Theorem 1.5. In $\S$ we introduce a morphism, weaker than a continuous map, suitable for pulling back systolic freedom. In $\$ 8$, we prove Theorem 1.1 and Theorem 1.4 . In $\$ 9$, we prove a refinement of Theorem 1.6 for the the 2-systole. In $\$ 10$, we establish the freedom of odd-dimensional systoles.

The paper concludes with 5 appendices. In Appendix A, we describe the techniques used in proving systolic freedom in the context of a pair of distinct complementary dimensions. In Appendix B, we prove a workhorse lemma, which allows us to propagate systolic freedom. In Appendix $\mathrm{Q}$, we prove a lemma (used in paragraph 7.2) on surgeries along curves in 4-manifolds. In Appendix D, we use the technique of spinning to identify explicitly the result of a useful surgery. In Appendix E, we recall the necessary material on Whitehead products and Hilton's theorem on homotopy groups of bouquets of spheres. 
Acknowledgments. The first author is grateful to Misha Farber for the opportunity to present an early version of this paper at the Topology conference at Tel Aviv University in June of 1998, and to Shmuel Weinberger for a helpful discussion of the results herein.

\section{ISOPERIMETRIC INEQUALITIES AND STABLE SYSTOLES}

2.1. Isoperimetric inequality. Every simple closed curve in the plane satisfies the inequality

$$
\frac{A}{\pi} \leq\left(\frac{L}{2 \pi}\right)^{2}
$$

where $L$ is the length of the curve and $A$ is the area of the region it bounds. This classical isoperimetric inequality is sharp insofar as equality is attained only by a round circle.

2.2. Loewner's theorem. In the 1950's, C. Loewner and P. Pu proved the following two theorems.

Let $\mathbb{R} \mathbb{P}^{2}$ be the real projective plane endowed with an arbitrary metric, i.e., an embedding in some $\mathbb{R}^{n}$. Then

$$
\left(\frac{L}{\pi}\right)^{2} \leq \frac{A}{2 \pi}
$$

where $A$ is its total area and $L$ is the length of its shortest non-contractible loop. This isosystolic inequality is also sharp, to the extent that equality is attained only for a constant curvature metric (quotient of a round sphere).

Similarly, every metric torus $T^{2}=\mathbb{R}^{2} / \mathbb{Z}^{2}$ satisfies the sharp inequality

$$
L^{2} \leq \frac{2}{\sqrt{3}} A,
$$

where $L$ is the length of its shortest non-contractible loop, and $A$ is the area.

2.3. What is a systole? In the 1970's, Marcel Berger initiated the study of a new Riemannian invariant, which eventually came to be called the systole.

Definition 2.1. The (homology) 1-systole of a Riemannian manifold $(X, g)$ is the quantity

$$
\operatorname{sys}_{1}(g)=\inf _{\ell} \operatorname{length}(\ell),
$$

where the infimum is taken over all closed curves $\ell$ in $X$ which are not homologous to zero.

A similar homotopy invariant, $\pi$-sys ${ }_{1}$, is obtained by minimizing lengths of noncontractible curves. These two invariants obviously coincide for $\mathbb{R P}^{2}, T^{2}$, and any manifold with abelian fundamental group.

2.4. Conformal representation and Cauchy-Schwartz. We give a slightly modified version of M. Gromov's proof of Loewner's theorem for the 2-torus, or rather the following slight generalization. 


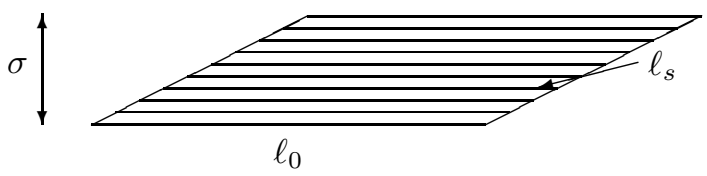

FIgURE 1. Family of parallel geodesics on a flat torus

Theorem 2.2. There exists a pair of distinct closed geodesics on an arbitrary metric 2-torus $T$, of respective lengths $L_{1}$ and $L_{2}$, such that

$$
L_{1} L_{2} \leq \frac{2}{\sqrt{3}} A,
$$

where $A$ is the total area of the torus. Equality is attained precisely (up to scaling) for the equilateral torus $(1, \zeta)$, where $\zeta$ is a primitive sixth root of unity. Moreover, their homotopy classes form a generating set for $\pi_{1}(T)=\mathbb{Z} \times \mathbb{Z}$.

Proof. A conformal representation $\phi: T_{0} \rightarrow T$, where $T_{0}$ is flat, may be chosen in such a way that $T$ and $T_{0}$ have the same area. Let $f$ be the conformal factor of $\phi$.

Let $\ell_{0}$ be any closed geodesic in $T_{0}$. Let $\left\{\ell_{s}\right\}$ be the family of geodesics parallel to $\ell_{0}$, see Figure 1. Parametrize the family $\left\{\ell_{s}\right\}$ by a circle $S^{1}$ of length $\sigma$ so that $\sigma \ell_{0}=\operatorname{area}\left(T_{0}\right)$. Then $\operatorname{area}(T)=\int_{T_{0}} \mathrm{Jac}_{\phi}=\int_{T_{0}} f^{2}$. By Fubini's theorem, $\operatorname{area}(T)=\int_{S^{1}} d s \int_{\ell_{s}} f^{2} d t$. By the Cauchy-Schwartz inequality,

$$
\operatorname{area}(T) \geq \int_{S^{1}} d s \frac{\left(\int_{\ell_{s}} f d t\right)^{2}}{\ell_{0}}=\frac{1}{\ell_{0}} \int_{S^{1}} d s\left(\text { length } \phi\left(\ell_{s}\right)\right)^{2} .
$$

Hence there is an $s_{0}$ such that $\operatorname{area}(T) \geq \frac{\sigma}{\ell_{0}}$ length $\phi\left(\ell_{s_{0}}\right)^{2}$, i.e.,

$$
\text { length } \phi\left(\ell_{s_{0}}\right) \leq \ell_{0} \text {. }
$$

This reduces the proof to the flat case. Given a lattice in $\mathbb{C}$, we choose a shortest lattice vector $L_{1}$, as well as a shortest one $L_{2}$ not proportional to $L_{1}$. The inequality is now obvious from the geometry of the standard fundamental domain in $\mathbb{C}$.

Theorem 2.3 (Gromov [12]). Let $X^{n}$ be a compact, orientable, smooth manifold of dimension $n$. Let $\pi$-sys ${ }_{1}(g)$ be the length of the shortest non-contractible loop for the metric $g$ on $X$. Then the inequality

$$
\left(\pi \text { - } \text { sys }_{1}(g)\right)^{n} \leq \text { Const } \cdot \operatorname{vol}(g)
$$

holds for a positive constant Const independent of the metric, if an only if the inclusion of $X$ in an Eilenberg-MacLane space $K=K\left(\pi_{1}(X), 1\right)$ retracts to the $(n-1)$-skeleton of $K$. For manifolds satisfying (1), moreover, the constant Const $=$ Const $_{n}$ depends only on $n=\operatorname{dim} X$.

The converse of this theorem was clarified by I. Babenko [1] (cf. Appendix B, where similar techniques are used). Note that the class of manifolds satisfying inequality (11) includes aspherical manifolds, as well as real projective spaces. 
2.5. Higher systolic invariants. Let $X$ be a finite $n$-dimensional simplicial complex endowed with a piecewise smooth metric $g$. Let $k \leq n$.

Definition 2.4. Let $\alpha \in H_{k}(X ; \mathbb{Z})$. Define

$$
\|\alpha\|=\inf _{M \in \alpha} \operatorname{vol}_{k}(M),
$$

where the infimum is taken over all piecewise smooth cycles $M$ representing the class $\alpha$. Here the volume of a (smooth) singular simplex is that of the pullback of the quadratic form $g$ to the simplex, and we take absolute values of the coefficients to obtain the volume of the cycle. Also define a 'stable norm' by

$$
\|\alpha\|_{s}=\lim _{q \rightarrow \infty} \frac{\|q \alpha\|}{q}
$$

Clearly, we have $\|\alpha\|_{s} \leq\|\alpha\|$.

Definition 2.5. We define the following three systolic invariants for the metric $g$ on $X$ :

(a) The ordinary systole

$$
\operatorname{sys}_{k}(g)=\inf _{\alpha \neq 0}\|\alpha\|
$$

(b) The systole 'modulo torsion'

$$
\operatorname{sys}_{k}^{\infty}(g)=\inf _{\alpha \neq \text { torsion }}\|\alpha\| .
$$

(c) The stable systole

$$
\operatorname{stabsys}_{k}(g)=\inf _{\alpha \neq \text { torsion }}\|\alpha\|_{s} .
$$

Evidently, we have $\operatorname{stabsys}_{k}(g) \leq \operatorname{sys}_{k}^{\infty}(g)$.

2.6. Calibration and stable systolic inequalities. Higher systolic invariants can sometimes also be constrained by the volume. Such constraint is illustrated by the following theorem.

Theorem 2.6 (Gromov [15]). For every metric $g$ on $\mathbb{C P}^{n}$,

$$
\operatorname{stabsys}_{2}^{n}(g) \leq K_{n} \operatorname{vol}_{2 n}(g),
$$

for a suitable constant $K_{n}$.

Proof. The proof is a calibration argument. Let $\alpha=\left[\mathbb{C P}^{1}\right]$ be the standard generator of $H_{2}\left(\mathbb{C P}^{n} ; \mathbb{Z}\right)=\mathbb{Z}$. Let $\eta \in H^{2}\left(\mathbb{C P}^{n} ; \mathbb{Z}\right)$ be the dual generator. Let $\omega$ be any 2 -form representing $\eta$. Then

$$
1=\int_{\mathbb{C P}^{n}} \omega^{\wedge n} \leq K\|\omega\|^{n} \operatorname{vol}_{2 n}(g)
$$

Hence $1 \leq K\|\eta\|^{n} \operatorname{vol}_{2 n}(g)=K \frac{\operatorname{vol}_{2 n}(g)}{\|\alpha\|_{s}^{n}}$, since the norms \|\| in $H^{2}(X)$ and \|\|$_{s}$ in $H_{2}(X)$ are dual by [9], p. 394 .

Thus $\|\alpha\|_{s}^{n} \leq K \operatorname{vol}_{2 n}(g)$, and so $\operatorname{stabsys}_{2}^{n}(g) \leq K \operatorname{vol}_{2 n}(g)$, a stable systolic inequality. 
Another example (also due to Gromov) of a stable systolic inequality is the following. Let $X=S^{p} \times S^{q}$ endowed with an arbitrary metric $g$. Then

$$
\operatorname{stabsys}_{p}(g) \cdot \operatorname{stabsys}_{q}(g) \leq \operatorname{vol}_{p+q}(g) \text {. }
$$

J. Hebda proved further results of this type in the 1980's.

Our objective is to show that in general, $k$-systoles are not constrained by the volume, unlike the above theorems for $T^{2}, \mathbb{R P}^{2}$, and $\operatorname{stabsys}_{2}\left(\mathbb{C P}^{n}\right)$.

\section{History AND DEFINITIONS}

3.1. Question of freedom or constraint. M. Berger asked in 1972 if the systolic invariants can be constrained by the volume. M. Gromov reiterated this question in [17]:

Question 3.1. Can one replace stable systoles by ordinary ones in the above inequalities?

The question was asked again in an IHES preprint in 1992 (which ultimately appeared as [14). Shortly afterwards, Gromov described the first example of systolic freedom, i.e., the violation of the systolic inequalities (see paragraph 3.2). The educated guess today is that, if one uses integer coefficients, systolic freedom predominates as soon as one is dealing with a $k$-systole for $k \geq 2$ (but see Remark 1.3).

3.2. Gromov's example. Gromov described metrics on $S^{1} \times S^{3}$ which provided an unexpected negative answer to the question 3.1. He stated it in global Riemannian terms. We provide a reformulation in terms of differential forms, which lends itself easier to generalization.

Let $b=\left.J(d r)\right|_{T S^{3}}$ be the contact 1 -form on $S^{3}$, where $S^{3} \subset \mathbb{C}^{2}$ is the unit sphere, $J$ is rotation by $\pi / 2$ furnished by the complex structure, $d r$ is the 1 -form on $\mathbb{C}^{2} \backslash\{0\}$, defined by the radial coordinate $r$.

Complete $b$ to an orthonormal basis $\left\{b, b^{\prime}, b^{\prime \prime}\right\}$ of $T^{*} S^{3}$, which is canonically identified with $T S^{3}$ by means of the standard unit sphere metric.

Let $S^{1}$ be the unit circle parametrized by $e^{i z}$, where $z$ is real. The standard 1 -form $d z$ is the arc-length. Gromov's sequence of left-invariant metrics, $\left\{g_{j}\right\}_{j=1}^{\infty}$, on $S^{1} \times S^{3}$ is defined by the following quadratic forms:

$$
g_{j}=(d z-j b)^{2}+b^{2}+\left(1+j^{2}\right)\left({b^{\prime 2}}^{2}+b^{\prime \prime 2}\right) .
$$

The essential ingredient here is the matrix

$$
\left[\begin{array}{cc}
1 & -j \\
-j & 1+j^{2}
\end{array}\right] \leftarrow z
$$

The coefficient $1+j^{2}$ ultimately determines the 3 -systole, the coefficient 1 determines the 1-systole, and the determinant of the matrix determines the volume of $g_{j}$. Then we obtain

$$
\frac{\operatorname{vol}\left(g_{j}\right)}{\operatorname{sys}_{1}\left(g_{j}\right) \operatorname{sys}_{3}\left(g_{j}\right)} \underset{j \rightarrow \infty}{\longrightarrow} 0 .
$$

See Appendix A for a 'local' version of this example, suitable for generalization.

Definition 3.2. A finite $n$-dimensional CW-complex $X$ is $m$-systolically free if

$$
\inf _{g} \frac{\operatorname{vol}_{n}(g)^{\frac{m}{n}}}{\operatorname{sys}_{m}(g)}=0
$$


where the infimum is taken over all piecewise smooth metrics $g$ on a simplicial complex $X^{\prime}$ in the homotopy type of $X$.

Remark 3.3. That every finite $\mathrm{CW}$-complex $X$ is homotopy equivalent to a finite simplicial complex $X^{\prime}$ is well-known, see [21]. That the definition does not depend on the choice of $X^{\prime}$ follows from Corollary 7.11 below. The systolic freedom of $X$ is equivalent to the existence of an $m$-free sequence of metrics, $\left\{g_{j}\right\}$, on $X^{\prime}$ :

$$
\operatorname{sys}_{m}^{n}\left(g_{j}\right) \geq j \operatorname{vol}_{n}^{m}\left(g_{j}\right) .
$$

Definition 3.4. An $n$-dimensional CW-complex is m-systolically free (modulo torsion) if

$$
\inf _{g} \frac{\operatorname{vol}_{n}(g)^{\frac{m}{n}}}{\operatorname{sys}_{m}^{\infty}(g)}=0
$$

Theorem 1.4 in the Introduction uses the following terminology in the context of coefficients modulo 2 .

Definition 3.5. Let $X$ be a compact, smooth manifold of dimension $n$. Consider $m$-cycles with $\mathbb{Z}_{2}$-coefficients, e.g., maps of manifolds (orientable or not) into $X$, which represent non-trivial classes in $H_{m}\left(X ; \mathbb{Z}_{2}\right)$, and calculate the $m$-volume of the pullback metric. Define $\mathbb{Z}_{2}$-sys $m(X)$ to be the infimum of all such volumes. We say that $X$ is $m$-systolically free over $\mathbb{Z}_{2}$ if

$$
\inf _{g} \frac{\operatorname{vol}_{n}(g)^{\frac{m}{n}}}{\mathbb{Z}_{2}-\mathrm{Sys}_{m}(g)}=0
$$

\section{Reduction to LOop SPACE}

For even $m$, we reduce the systolic problem to spaces each of whose Betti numbers is at most 1 . More specifically, we show that the systolic $m$-freedom of $n$-manifolds (modulo torsion) reduces to that of certain skeleta of loop spaces of spheres. Thus, whether or not there exists an analogue of Gromov's Theorem 2.3 for the higher systoles, depends on the loop space in question.

Our proof of Theorem 1.6 in the Introduction is modeled on the proof of Lemma 4.2 below.

Lemma 4.1. Let $m \geq 1$ be an integer. Let $X$ be a finite $C W$-complex. Let $b=b_{m}(X)$. Let $K=K\left(\mathbb{Z}^{b}, m\right)$ be an Eilenberg-MacLane space with skeleta $K^{(m+1)}=K^{(m)}=\vee^{b} S^{m}$. There is a map $X \rightarrow K$, inducing an isomorphism $H_{m}(X, \mathbb{Z}) /$ torsion $\rightarrow H_{m}(K, \mathbb{Z})$, and whose restriction to $X^{(m+1)}$ has image in $\vee^{b} S^{m}$.

Proof. A basis for $H^{m}(X ; \mathbb{Z}) /$ torsion defines a map $X \rightarrow K$, inducing the required isomorphism. The cellular approximation theorem yields a map $X^{(m+1)} \rightarrow \vee^{b} S^{m}$.

Lemma 4.2. Any $(2 m-1)$-dimensional $C W$-complex $X$ admits a map to a suitable bouquet of $m$-spheres which induces an isomorphism in rational homology of dimension $m$. 
Proof. Consider the bouquet $\vee^{b} S^{m}$, where $b=b_{m}(X)$. According to B. Eckmann [8], a map $\phi_{q}: S^{m} \rightarrow S^{m}$ of degree $q$ induces multiplication by $q$ in the stable groups $\pi_{j}\left(S^{m}\right)$, for $m+1 \leq j \leq 2 m-2$. Thus, if $q$ is a multiple of the order of $\pi_{j}\left(S^{m}\right)$, the self-map $\vee^{b} \phi_{q}: \vee^{b} S^{m} \rightarrow \vee^{b} S^{m}$ induces the zero homomorphism in $\pi_{j}$ by Hilton's theorem E.1. Hence a map $f_{j}: X^{(j)} \rightarrow \vee^{b} S^{m}$, followed by $\vee^{b} \phi_{q}$, extends to $f_{j+1}: X^{(j+1)} \rightarrow \vee^{b} S^{m}$. The lemma now follows by induction on $j$, based on Lemma 1.1 .

Corollary 4.3. Let $m$ and $n$ be integers such that $2 \leq m<n<2 m$. Then every $n$-dimensional manifold is m-systolically free (modulo torsion).

Proof. The bouquet of $m$-spheres, viewed as an $n$-dimensional complex, is obviously $m$-free (the numerator in Definition 3.2 vanishes). We apply the pull-back Lemma B.2.

Lemma 4.4. The $(2 m-1)$-skeleton of a $(2 m)$-manifold $X$ admits a map to a suitable bouquet of $m$-spheres, inducing an isomorphism in $m$-dimensional rational homology, and sending the attaching maps of the $(2 \mathrm{~m})$-cells to a sum of Whitehead products.

Proof. We continue with the notation of the proof of Lemma 4.2. We thus have a map $f_{2 m-1}: X^{(2 m-1)} \rightarrow \vee^{b} S^{m}$. Let $e=\left[S^{m}\right]$ be the fundamental class of the sphere. Recall that the Whitehead product $[e, e] \in \pi_{2 m-1}\left(S^{m}\right)$ generates precisely the kernel of the suspension homomorphism. Suspension commutes with $\phi_{q}$. Hence, if $q$ is a multiple of the order of the stable group $\pi_{2 m}\left(S^{m+1}\right)$, then $\left(\phi_{q}\right)_{*}\left(\pi_{2 m-1}\left(S^{m}\right)\right)$ is contained in the subgroup generated by Whitehead products. Hence $\left(\vee^{b} \phi_{q}\right) \circ$ $f_{2 m-1}$ is the desired map.

Proof of Theorem 1.0. Consider the based loop space $L=\Omega\left(S^{m+1}\right)$. As shown by J.-P. Serre [24, all homotopy groups of $L$ are finite, except $\pi_{m}$ (here we need $m$ even). Let $q$ be the product of the orders of the homotopy groups of $L$ from $m+1$ up to dimension $n$. Let $\psi: L \rightarrow L$ be the map that sends a loop $\omega$ to $\omega^{q}$. Since the usual group structure on $\pi_{i}(L)$ coincides with the one coming from the multiplication of loops in $L$, the map $\psi$ induces multiplication by $q$ in each homotopy group of $L$. Thus, $\psi=0$ on $\pi_{i}(L)$, for $m+1 \leq i \leq n$.

Now let $X$ be an $n$-manifold, and let $b=b_{m}(X)$. Let $L^{\times b}$ be the Cartesian product of $b$ copies of $L$. With respect to a suitable cell structure, the $m$-skeleton of $L^{\times b}$ is a bouquet $\vee^{b} S^{m}$ of $b$ copies of $S^{m}$. By Lemma 4.1, the $(m+1)$-skeleton of $X$ admits a map $f_{m+1}$ to the $m$-skeleton of $L^{\times b}$ which induces rational isomorphism in $m$-dimensional homology.

We now proceed as in the proof of Lemma 4.2, with the bouquet of spheres replaced by $L^{\times b}$. By induction, the map $\psi \circ f_{k}$ extends to

$$
f_{k+1}: X^{(k+1)} \rightarrow L^{\times b}
$$

for all $k$ from $m+1$ to $n$. In this fashion, we obtain a map $f: X \rightarrow L^{\times b}$ such that $f_{*}: H_{m}(X ; \mathbb{Q}) \rightarrow H_{m}\left(L^{\times b} ; \mathbb{Q}\right)$ is an isomorphism. By the pull-back Lemma B.2. the freedom of $X$ follows from that of $L^{\times b}$. It remains to reduce the freedom of $L^{\times b}$ to that of $L$ itself.

A well-known result of I. James (see [28]), states that $L$ has, up to homotopy, a cell decomposition

$$
L=S^{m} \cup e^{2 m} \cup e^{3 m} \cup \cdots
$$


with precisely one cell in each dimension divisible by $m$. Therefore, we may proceed as in $\$$, and carve up the $n$-skeleton of $L^{\times b}$, reducing the problem to the freedom of the closures of $n$-dimensional cells of $L$. Each such closure is a product of skeleta of $L$. Therefore, Theorem 1.6 results from the following lemma.

Lemma 4.5. Let $m \geq 2$. Then the product of an $(m-1)$-connected, $m$-systolically free complex with $S^{m}$ is again $m$-free. Furthermore, the product of $(m-1)$ connected, $m$-systolically free complexes is again $m$-free.

Proof. Given $m$-free families of metrics on each factor, we scale them to unit $m$ systole. We also scale the $m$-sphere to unit $m$-volume. The product metrics then form $m$-free families.

\section{Carving-up procedure}

The procedure in question is helpful in breaking up the problem of systolic freedom of complicated spaces into simpler components. We introduce it here as a way of streamlining the arguments of [3], where the authors, in collaboration with I. Babenko, proved the middle-dimensional systolic freedom of even-dimensional manifolds of dimension $n=2 m \geq 6$ with free $H_{m}(X, \mathbb{Z})$.

Let $K$ be an $n$-dimensional CW-complex with no cells in dimension $n-1$. The carving-up procedure consists of inserting a cylinder between each $n$-dimensional cell and its boundary. The new complex $K^{\prime}$ has the following three properties.

1. $K^{\prime}$ has the same homotopy type as $K$.

2. $K^{\prime}$ has the same number of top-dimensional cells $e_{i}^{n}$ as $K$.

3. The closures $\bar{e}_{i}^{n}$ of all top-dimensional cells in $K^{\prime}$ are disjoint.

Assume for the sake of simplicity that the image of each attaching map

$$
f_{i}: \partial D^{n} \rightarrow K^{(n-2)}
$$

of $e_{i}$ is a subcomplex (i.e., there are no partly covered cells in the $(n-2)$-skeleton). Let

$$
\partial^{i}=\operatorname{Im}\left(f_{i}\right) \subset K^{(n-2)}
$$

be the boundary of the $i^{\text {th }}$ top-dimensional cell.

The procedure of inserting cylinders in $K$ is formalized as follows:

$$
K^{\prime}=K^{(n-2)} \cup_{g_{i}^{0}}\left(\partial^{i} \times[0,1]\right) \cup_{g_{i}^{1}} \bar{e}_{i}^{n} .
$$

Here $K^{(n-2)}$ is the $(n-2)$-skeleton, and the extremities of the $i^{\text {th }}$ cylinder are attached along the inclusion maps $g_{i}^{0}: \partial^{i} \times\{0\} \rightarrow K^{(n-2)}$, and $g_{i}^{1}: \partial^{i} \times\{1\} \rightarrow \bar{e}_{i}^{n}$. The repeated upper and lower index $i$ indicates that the procedure is repeated for each top-dimensional cell, as in Einstein notation. Thus, we insert as many cylinders as there are top-dimensional cells, to obtain $K^{\prime}$.

Lemma 5.1. Let $K$ be an n-dimensional $C W$-complex with no cells in dimension $n-1$. Then the systolic $q$-freedom of $K$ reduces to that of the closures of its topdimensional cells.

Proof. We replace $K$ by $K^{\prime}$ as above. To make sure that areas and volumes are well-defined, we replace each $\partial^{i}$ by a simplicial complex of the same dimension and in the same homotopy type. Then the positive codimension condition is satisfied. We also replace each cell closure by a simplicial complex in the same homotopy 
type so as to extend the simplicial structure on $\partial^{i}$. We make a similar replacement for $K^{(n-2)}$, obtaining a simplicial complex $K^{\prime \prime}$.

We apply to $K^{\prime \prime}$ the long cylinder argument in the proof of Lemma B.1 and Lemma B.4. The key tools here are the isoperimetric inequality and the coarea inequality, applied to $\partial^{i} \times[0,1]$, where the metric on the interval is chosen sufficiently long, to minimize interaction between opposite extremes of the cylinder.

\section{MidDle-Dimensional FREEDOM}

We now establish systolic freedom (modulo torsion) in the middle dimension, as stated in Theorem 1.5. In fact, we show that the theorem is valid in the more general case of triangulable spaces with piecewise smooth metrics, for which of course areas and volumes can still be defined.

In contrast with Loewner's theorem 2.2, the middle-dimensional systole $(\mathrm{m}$ systole) is not constrained by the volume when $m \geq 2$. Thus Loewner's theorem has no higher-dimensional analogue if one works with orientable submanifolds. On the other hand, there might be such an analogue if one allows non-orientable submanifolds (see Remark 1.3).

A proof in the case $m \geq 3$ appeared in [3].

6.1. Idea of proof. The idea of the proof of Theorem 1.5 is to reduce the problem to a local version of Gromov's example, described in \$3.2, by using pullback arguments of Appendix B as follows. High-degree self-maps of $S^{m}$ combined with Hilton's theorem E.1 allow us to map $X$ to a kind of a first-order approximation to the $2 m$-skeleton of the Eilenberg-MacLane space $K\left(\mathbb{Z}^{b}, m\right)$. This approximation has the same rational homology and contains the minimal number of $2 m$-dimensional cells. The carving-up procedure of $\$ 5$ reduces the problem to the freedom of the closures of these cells, each with middle Betti number at most 2. An additional pull-back reduces the problem to the freedom of $S^{m} \times S^{m}$. Finally, the latter is reduced to the $(1, m)$-freedom of $S^{1} \times S^{m}$ (cf. Appendix A).

6.2. Proof of Theorem 1.5. Let us restate the theorem in a more convenient (and slightly more general) setting.

Theorem 6.1. Let $m \geq 2$. Let $X$ be a finite, triangulable $C W$-complex of dimension $2 m$. Then $X$ is m-systolically free (modulo torsion).

Proof. Let $b=b_{m}(X)$. Let $P=S^{m} \cup_{[e, e]} D^{2 m}$, where $e$ is the fundamental class of $S^{m}$. Consider the Cartesian product $Q=P^{\times b}$ of $b$ copies of $P$. Its $m$-skeleton $Q^{(m)}=\vee^{b} S^{m}$ is a bouquet of $b$ copies of $S^{m}$. Let $f: X^{(2 m-1)} \rightarrow Q^{(m)}$ be the map given by Lemma 4.4. Here the image of $\pi_{2 m-1}\left(X^{(2 m-1)}\right)$ is contained in the subgroup generated by Whitehead products. Then $f$, followed by the inclusion $Q^{(m)} \hookrightarrow Q^{(2 m)}$, extends across all of $X$ by Hilton's theorem E.1. Thus the freedom of $X$ reduces to that of the $(2 m)$-skeleton of $P^{\times b}$.

We now apply Lemma 5.1 to obtain a further reduction to only two cases: $S^{m} \times$ $S^{m}$ and $P$ itself. The CW-complex $P$ is homotopy equivalent to the regular CWcomplex $W=S^{m} \times S^{m} \cup_{a+b} D^{m+1}$ (product of spheres with a disk attached along the diagonal). Thus the systolic $m$-freedom of $P$ reduces to that of $S^{m} \times S^{m}$ by Lemmas B.1 and B.2.

Finally, the middle-dimensional freedom of $S^{m} \times S^{m}$ follows from Lemmas 6.2 and 6.3 below. 
Lemma 6.2. Let $m \geq 2$. Then the manifold $S^{m} \times S^{m}$ admits a map to a $C W$ complex obtained from $S^{1} \times S^{m-1} \times S^{m}$ by attaching cells of dimension at most $2 m-1$, which induces a monomorphism in $m$-dimensional homology.

Proof. The manifolds $S^{m}$ and $S^{1} \times S^{m-1}$ are related by surgery, and thus $S^{m} \times S^{m}$ admits a map to $S^{1} \times S^{m-1} \times S^{m} \cup D^{2} \times * \times S^{m}$.

For $m=2$, this does not define a meromorphic map, since we have added top dimensional cells. In this case, we proceed as in Corollary 7.9.

Lemma 6.3. The manifold $S^{1} \times S^{m-1} \times S^{m}$ is $m$-systolically free if $m \geq 2$.

Proof. The manifold $S^{1} \times S^{m}$ is $(1, m)$-free by Theorem A.2. Let $g_{j}$ be such a free sequence of metrics on $S^{1} \times S^{m}$. Taking the product with a sphere $S^{m-1}$ of volume equal to $\frac{\operatorname{sys}_{m}\left(g_{j}\right)}{\operatorname{sys}_{1}\left(g_{j}\right)}$, we obtain an $m$-free sequence of metrics on $S^{1} \times S^{m-1} \times S^{m}$.

6.3. Spin manifolds. Our Theorem 1.1 improves the general middle-dimensional result in the case $m=2$, to the extent that it removes the 'modulo torsion' clause. A similar improvement exists for $m=3$ and $m=4$.

Proposition 6.4. Spin manifolds of dimension 6 and 8 are systolically free in middle dimension.

Proof. We reduce the problem to the simply-connected case as in paragraph 8.2 . Furthermore, the spin condition $w_{2}=0$ ensures that all embedded 2-spheres have trivial normal bundles. Let $C$ be the union of a disjoint family of embedded 2spheres representing a set of generators for 2-dimensional homology, and perform surgery along $C$. An analogue of Lemma 7.5 reduces the problem to the 2-connected case. A 2-connected 6-manifold has torsion-free 3-dimensional homology, and the proposition is established for a 6 -manifold.

For 8-manifolds, we continue by choosing a disjoint family of embedded 3spheres (whose normal bundles are automatically trivial) which represent generators for 3-dimensional homology, and argue as before, reducing the problem to the 3 -connected case.

\section{MEROMORPHIC MAPS}

Here we develop a convenient language for establishing the 2-systolic freedom of orientable 4-manifolds. We define a morphism, weaker than a continuous map, suitable for pulling back systolic freedom. Our technique is introduced most provocatively by means of the following question.

Question 7.1. What do meromorphic maps and surgeries have in common?

The answer is, roughly speaking, as follows (see Example 7.4 and Lemma 7.5): both of them carry an underlying structure, crystallized in the concept of a 'topological meromorphic map' below.

7.1. 'Topological meromorphic maps'. Here we attempt to define a morphism, weaker than a continuous map, suitable for pulling back systolic freedom. Such morphisms could also be called 'topological blow-up maps' or 'topological rational maps'. 
Definition 7.2. Let $X^{n}$ and $Y^{n}$ be manifolds of dimension $n=2 m$. A "meromorphic map", denoted by a broken arrow below,

$$
X-\rightarrow Y
$$

is a continuous map $f: X \rightarrow W$, such that:

(a) The space $W$ has the homotopy type of a CW-complex obtained from $Y$ by attaching cells of strictly smaller than the top dimension: $W \simeq Y \cup \bigcup_{i} e_{i}^{\leq n-1}$.

(b) The map $f$ induces a monomorphism in the middle dimension: $\operatorname{ker}\left(f_{*}\right.$ : $\left.H_{m}(X) \rightarrow H_{m}(W)\right)=0$.

Remark 7.3. We do not require the inclusion $Y \rightarrow W$ to induce an epimorphism in middle-dimensional homology.

Example 7.4. Let $X$ be a complex manifold of (complex) dimension $m$, let $\widehat{X} \rightarrow X$ be the blow-up at a point $p \in X$, and let $\phi: X-\rightarrow \widehat{X}$ be the classical meromorphic map (undefined at $p$ ). For $m=2$, set $W=\widehat{X} \cup D^{3}$, where the 3-cell is attached along the exceptional curve $S^{2}$. Then $\phi$ can be modified in a neighborhood $D^{4}$ of $p$ so as to lift a continuous map $f: X \rightarrow W$ (a homotopy equivalence). Here we take the cone $D^{4} \rightarrow D^{3}$ of the Hopf fibration $S^{3} \rightarrow S^{2}$. More precisely, the Hopf fibration extends to a continuous map whose restriction to the complement of $D^{4}$ in $X$ is a diffeomorphism onto the complement of $S^{2}$ in $\widehat{X}$, while $D^{4}$ is mapped to $D^{3}$ as described. For general $m$ we have a continuous map $X \rightarrow \widehat{X} \cup \operatorname{Cone}\left(\mathbb{C P}^{m-1}\right)$.

7.2. Surgery and meromorphic maps. We now show that, under certain homological conditions, surgeries along curves yield meromorphic maps between 4manifolds.

Lemma 7.5. Let $X$ be a closed, orientable, smooth 4-manifold. Let $C \subset X$ be a union of smoothly embedded, disjoint closed curves. Let $Y$ be the result of surgery along $C$. Then $X$ admits a meromorphic map to $Y$. The conclusion holds equally well if one uses coefficients modulo 2 in the definition of homology and meromorphic maps.

Proof. Since $X$ is orientable, the normal disk bundle of $C$ is trivializable. Over each component of $C$, there are two possible trivializations (or, framings), corresponding to $\pi_{1}(\mathrm{SO}(3))=\mathbb{Z}_{2}$. The framing (which is part of the surgery data) identifies the normal disk bundle with $C \times D^{3}$. By definition,

$$
Y=\left(X \backslash C \times \operatorname{int} D^{3}\right) \cup \bigcup_{i}\left(D_{i}^{2} \times S^{2}\right),
$$

where $\bigcup_{i} D_{i}^{2}$ is a disjoint union of 2-disks (one for each connected component $C_{i}$ of $C$ ). Let $Z=X \times I \cup \bigcup_{i} D_{i}^{2} \times D^{3}$ be the result of attaching a handle of index 2 to $X$ along each component of $C$, according to the given framing. Then $Z$ is the cobordism between $X$ and $Y$ determined by the surgery, see J. Milnor [22].

Now let $W=X \cup \bigcup_{i} D_{i}^{2}$ be the mapping cone of the inclusion $C \subset X$. In other words, $W$ is the CW-complex obtained from $X$ by attaching the cores of the handles.

Clearly, the inclusion of $X$ in $W$ induces a monomorphism in 2-dimensional homology. Moreover, $W$ is a deformation-retract of $Z$, see [22]. Figure 2 depicts the various spaces introduced so far. 


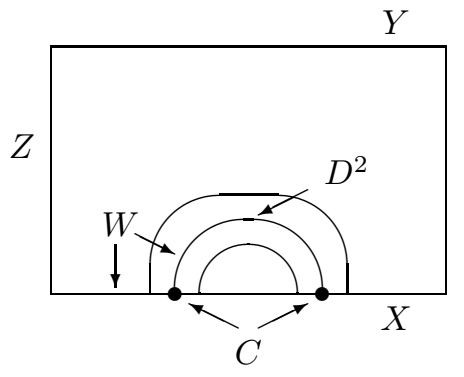

FiguRE 2. Surgery and cobordism

We have an inclusion $i: X \subset Z$, as well as a homotopy equivalence $Z \simeq Y \cup e^{3}$. This gives a map $X \rightarrow Y \cup e^{3}$, which satisfies condition (b) in Definition 7.2 of injectivity in 2-dimensional homology, since $Z$ is homotopy equivalent to $W$.

Corollary 7.6. An orientable 4-manifold admits a meromorphic map to a simply connected one.

Proof. Let $C \subset X$ be the union of a family of disjoint embedded closed curves representing a set of generators for the fundamental group. Surgery on $X$ along $C$ produces a simply-connected manifold $Y$, and we apply Lemma 7.5 .

Of particular interest to us (cf. Corollary 7.9) is a 'dual' version of Lemma 7.5 (the meromorphic map goes the other way).

Proposition 7.7. Let $X$ be a closed, orientable, smooth 4-manifold. Let $Y$ be the result of surgery along a union $C$ of smoothly embedded, disjoint closed curves in $X$. Assume that the connected components of $C$ define a linearly independent set in $H_{1}(X ; \mathbb{Q})$. Then $Y$ admits a meromorphic map to $X$.

Proof. We continue with the notation of Lemma 7.5. Let $j: Y \rightarrow Z$ be the inclusion and $g: Z \rightarrow W$, the homotopy equivalence. Then $g_{*} \circ j_{*}: H_{2}(Y) \rightarrow H_{2}(W)$ is a monomorphism. This is established by induction on the number of connected components of $C$, using Lemma C.1 in Appendix Q.

More precisely, let $\psi$ be the isomorphism provided by Lemma C.1. Since $W$ is obtained from $X$ by attaching 2-cells, the inclusion $i: X \rightarrow Z$ induces a monomorphism $i_{*}: H_{2}(X) \rightarrow H_{2}(Z)$. Since $j_{*} \circ \psi=i_{*}$, it follows that $j_{*}$ is injective, and so $g_{*} \circ j_{*}$ is, too.

Hence, $g \circ j: Y \rightarrow W$ satisfies condition (b) in Definition 7.2. We thus have the required meromorphic map $Y \rightarrow \rightarrow X$.

Remark 7.8. Without the independence hypothesis, $Y$ may have a larger second Betti number than either $X$ or $W$. For example, if $X=S^{4}$ and $C=S^{1}$, then $W \simeq S^{2} \vee S^{4}$ and $Y=S^{2} \times S^{2}$ or $Y=S^{2} \widetilde{\times} S^{2} \cong \mathbb{C P}^{2} \# \overline{\mathbb{C P}}^{2}$, depending on the parity of the framing. Thus $b_{2}(X)=0, b_{2}(W)=1$, and $b_{2}(Y)=2$.

Corollary 7.9. There exists a meromorphic map $S^{2} \times S^{2}-\rightarrow S^{2} \times T^{2}$. 
Proof. Surgery on $T^{2} \times S^{2}$ along a pair of generators of $\pi_{1}\left(T^{2} \times S^{2}\right)=\mathbb{Z} \times \mathbb{Z}$ yields $S^{2} \times S^{2}$. An explicit verification of this fact is given in Corollary D.2. Alternatively, one readily sees that the surgery does not change the intersection form, and the resulting manifold is simply-connected. Hence it is certainly homotopy equivalent to $S^{2} \times S^{2}$. Either way, Proposition 7.7 applies, completing the proof.

7.3. Pullback lemma for free metrics. The following proposition allows us to propagate the phenomenon of freedom once we exhibit it for products of spheres.

Proposition 7.10. Let $f: X-\rightarrow Y$ be a meromorphic map. If $Y$ is systolically free, then so is $X$.

Corollary 7.11. Systolic freedom is a homotopy invariant.

Proof. Any map defining a homotopy equivalence is obviously a "meromorphic map" (here $W=Y$, i.e., the set of attached cells is empty).

Proposition 7.10 follows from the following lemma (cf. Lemma 6.1 of [2]).

Lemma 7.12. Let $X$ and $Y$ be triangulable $C W$-complexes of dimension $n$. Suppose there is a map $\phi: X \rightarrow W$ inducing a monomorphism on $H_{q}$, where $W=$ $Y \cup_{f} e^{k}$ is obtained from $Y$ by attaching a single cell of dimension $k \leq n-1$. Then the q-systolic freedom of $Y$ implies that of $X$. Moreover, if $X$ is a smooth manifold, the metrics exhibiting freedom may be chosen to be smooth.

The lemma is proved in Appendix B.

\section{SystoliC FREEDOM IN DIMENSION 4}

In this section, we show that orientable closed 4-manifolds are systolically free in the middle dimension.

In the simply-connected case, the first author, in collaboration with I. Babenko 21, already reduced the problem to the case of $S^{2} \times S^{2}$. Here we notice that $S^{2} \times S^{2}$ admits a meromorphic map to $S^{2} \times T^{2}$ (see Corollary 7.9), and so its middle-dimensional freedom results from that of $S^{2} \times T^{2}$ (see Lemma 6.3).

8.1. Systolic freedom modulo torsion. Prior to proving Theorem 1.1 of the Introduction, we establish freedom modulo torsion for an arbitrary simplicial complex of dimension 4 , as follows.

Theorem 8.1. Every compact, triangulable 4-dimensional $C W$-complex $X$ is 2systolically free (modulo torsion).

Proof. The map $X \rightarrow K\left(\mathbb{Z}^{b}, 2\right)$ of Lemma 4.1, followed by a homotopy equivalence $K\left(\mathbb{Z}^{b}, 2\right) \simeq\left(\mathbb{C P}^{\infty}\right)^{\times b}$ gives a map $i: X \rightarrow\left(\mathbb{C P}^{\infty}\right)^{\times b}$. Up to homotopy, we may assume that the image of $i$ lies in the 4 -skeleton $K_{b}=K\left(\mathbb{Z}^{b}, 2\right)^{(4)}$. By construction, the map $i$ induces an isomorphism in 2-dimensional homology with integer coefficients. By Lemma B.2, the systolic freedom of $X$ reduces to that of the 4-dimensional complex $K_{b}$.

The map to $K_{b}$ constructed in the proof of the theorem is only an isomorphism in rational 2-dimensional homology, so we have no control over areas of 2-cycles defining torsion classes.

The CW-complex $K_{b}$ contains no 3-cells. Indeed, it is obtained from the bouquet $\bigvee^{b} S_{r}^{2}$ of $b$ copies of $S^{2}$ by attaching 4-cells along all the Whitehead products 
$\frac{1}{2}\left[e_{r}, e_{r}\right]$ and $\left[e_{r}, e_{s}\right]$, where $e_{r}$ is the fundamental class of $S_{r}^{2}$ (cf. paragraph E.1). Therefore, the freedom of $K_{b}$ reduces to that of the closures of its 4-cells, by Lemma 5.1, since the isoperimetric inequality for small 1-cycles obviously holds for bouquets of spheres.

Each such closure is homeomorphic to either $\mathbb{C P}^{2}$ or $S^{2} \times S^{2}$. We are thus left with proving the systolic freedom of these two manifolds.

The freedom of $\mathbb{C P}^{2}$ reduces to that of the product of spheres as follows. Notice that there is a degree 4 map from $\mathbb{C P}^{2}$ to $P=S^{2} \cup_{[e, e]} D^{4}$, where $e$ is the fundamental class of $S^{2}$. Now $P$ is homotopy equivalent to $W=S^{2} \times S^{2} \cup_{a+b} D^{3}$, where $a$ and $b$ are the fundamental classes of the two factors. Thus $\mathbb{C P}^{2}$ admits a meromorphic map to $S^{2} \times S^{2}$, and we invoke Proposition 7.10 .

The product of spheres is systolically free by Corollary 7.9, Lemma 6.3, and Lemma B.2.

Corollary 8.2. Every compact, smooth 4-manifold $X$ admits metrics of arbitrarily small volume, with the following property: every orientable, immersed surface of smaller than unit area, defines a torsion class in $H_{2}(X, \mathbb{Z})$.

Proof. This is immediate from the theorem.

Note that the manifold $X$ in the above corollary may be non-orientable.

8.2. Orientable case. We now establish the systolic 2-freedom of orientable 4manifolds.

Proof of Theorem 1.1. Let $X$ be a compact, orientable, smooth 4-manifold. By Corollary 7.6 and Proposition 7.10, the freedom of $X$ reduces to that of a simplyconnected manifold $Y$. Since $Y$ is simply-connected, the group $H_{2}(Y ; \mathbb{Z})$ is free abelian. Hence the 2-freedom of $Y$ follows from Theorem 8.1.

8.3. The case of 2 -systolic freedom over $\mathbb{Z}_{2}$. The notion of freedom with coefficients modulo 2 was introduced in Definition 3.5. We now reduce the question of 2-systolic freedom over $\mathbb{Z}_{2}$ of arbitrary 4-manifolds to that of just two manifolds: $S^{2} \times S^{2}$ and $\mathbb{C P}^{2}$.

Proof of Theorem 1.4. The orientable case is reduced to the case when $X$ is simply connected, as in the proof of the previous theorem. The map to $K\left(\pi_{2}(X), 2\right)$ induces an isomorphism in 2-dimensional homology, whether with integer or mod 2 coefficients. Therefore the problem is further reduced to the freedom over $\mathbb{Z}_{2}$ of this Eilenberg-MacLane space. The long cylinder construction of Appendix B works equally well with $\mathbb{Z}_{2}$-coefficients. Thus we may carve up the space $K\left(\pi_{2}(X), 2\right)$ as above to reduce the problem to $\mathbb{C P}^{2}$ and $S^{2} \times S^{2}$.

The reduction of $\mathbb{C P}^{2}$ to $S^{2} \times S^{2}$ as in the proof of Theorem 8.1 does not work here, as the map $H_{2}\left(\mathbb{C P}^{2} ; \mathbb{Z}_{2}\right) \rightarrow H_{2}\left(S^{2} \cup_{[e, e]} D^{4} ; \mathbb{Z}_{2}\right)$ is not injective.

\section{Systolic 2-Freedom in arbitrary Dimension}

The absence of odd cells in a suitable decomposition of the Eilenberg-MacLane space $K(\mathbb{Z}, 2)=\mathbb{C P}^{\infty}$ is the key to the proof of Theorem 8.1.

The method employed in that proof can be used to improve Theorem 1.6 for $m=2$. We illustrate this by reducing the systolic 2 -freedom of all manifolds $X$ with torsion-free $H_{2}(X, \mathbb{Z})$ to that of a particularly simple list of manifolds. 
Theorem 9.1. The following two statements are equivalent:

(i) All compact, smooth manifolds $X$ with torsion-free $H_{2}(X)$ are 2-systolically free;

(ii) For each $n \geq 2$, the manifold $\mathbb{C P}^{n}$ is 2-systolically free.

Proof. The 2-freedom of each projective space would imply that of the product of arbitrarily many factors, as in Lemma 4.5. The 2 -freedom of a product of several copies of $\mathbb{C P}^{1}$ follows from Corollary 7.9. But each cell closure in the standard simplicial structure of $K\left(\mathbb{Z}^{b}, 2\right)$ is such a product, proving that any finite skeleton would also be 2 -free. We map $X$ to $K\left(\mathbb{Z}^{b}, 2\right)$ as in Lemma 4.1 and apply the pull-back Lemma B.2.

\section{ODD-DIMENSIONAL FREEDOM}

Note that the systolic $m$-freedom of $X$ when $m>\frac{n}{2}$ follows from the $m$-freedom of a bouquet of $m$-spheres viewed as an $n$-dimensional CW-complex, by Corollary 4.3. Thus the interesting case is $n>2 m$.

Theorem 10.1. Let $n$ and $m$ be integers satisfying $3 \leq m<n$, where $m$ is odd. Then every $n$-dimensional manifold $X$ is $m$-systolically free (modulo torsion).

Proof. The starting point is again the map from the $(m+1)$-skeleton of $X$ to the bouquet of spheres, as in Lemma 4.1. For odd $m$, the only non-trivial Whitehead products are the 'mixed' ones. Thus, high-degree self-maps of the sphere allow us to map $X$ to the product $\left(S^{m}\right)^{\times b}$ of $b=b_{m}(X)$ copies of $S^{m}$, as in the proof of Lemma 4.2 .

Here we rely upon the existence of self-maps inducing the zero homomorphism in every given homotopy group of the sphere. Indeed, according to D. Sullivan [26], a self-map of $S^{m}$ of degree $d$ induces a nilpotent map in the $d$-torsion of $\pi_{j}\left(S^{m}\right)$, for every $j>0$.

Now if $m$ does not divide $n$, the $n$-skeleton of $\left(S^{m}\right)^{\times b}$ coincides with the $(n-1)$ skeleton, in which case we actually get (singular) metrics on $X$ of vanishing $n$ volume.

If $m$ divides $n$, the $n$-skeleton of $\left(S^{m}\right)^{\times b}$ contains no $(n-1)$-cells, and we use the carving-up procedure of $\$ 5$ to reduce to products of spheres.

\section{Appendix A. Systoles of COMPlementary Dimensions}

To describe phenomena along the lines of Gromov's example, it is convenient to introduce the following terminology.

Definition A.1. A compact, smooth, $(p+q)$-dimensional manifold $X$ is called systolically $(p, q)$-free if

$$
\inf _{g} \frac{\operatorname{vol}_{p+q}(g)}{\operatorname{sys}_{p}(g) \operatorname{sys}_{q}(g)}=0,
$$

where the infimum is taken over all metrics $g$ on $X$.

We present a proof of $(1, n-1)$-freedom, originally obtained by the first author in collaboration with I. Babenko in [2].

Theorem A.2. Every compact, orientable, smooth manifold of dimension $n \geq 3$ is $(1, n-1)$-free. 
Proof. We obtain systolically free families of metrics on the manifold $X^{n}$ by a direct geometric construction. The idea is to introduce a local version of Gromov's example ( $c f .3(3.2)$, i.e., a metric on a manifold with boundary which can be glued into any manifold to ensure systolic freedom. Here the matrix of Gromov's example:

$$
\left[\begin{array}{cc}
1 & -j \\
-j & 1+j^{2}
\end{array}\right] \leftarrow z
$$

for a given $j$, is replaced by the matrix

$$
\left[\begin{array}{cc}
1 & -x \\
-x & 1+x^{2}
\end{array}\right] \leftarrow z
$$

where the coefficient $x$ varies between 0 and $j$.

Let $C$ be a union of closed curves which form a basis for $H_{1}(X ; \mathbb{Q})$. Its tubular neighborhood is diffeomorphic to $C \times D^{n-1}$. Let $K \subset D^{n-1}$ be a codimension 2 submanifold with trivial normal bundle (e.g., the $(n-3)$-sphere). The boundary of a tubular neighborhood of $C \times K$ is diffeomorphic to the hypersurface $\Sigma:=C \times K \times T^{1}$, where $T^{1}$ is a circle. Our construction is local in a neighborhood of $\Sigma$. Choose a fixed metric on $X$ satisfying the following four properties:

1. It is a direct product in a neighborhood of $\Sigma$.

2. The hypersurface $\Sigma$ is a metric direct product of the three factors $C, K$, and $T^{1}$.

3. Each connected component of $C$ is a circle of length 1 .

4. The circle $T^{1}$ has length 1 .

We now cut $X$ open along $\Sigma$ and insert suitable 'cylinders' $\Sigma \times I$, indexed by $j \in \mathbb{N}$, resulting in a sequence of metrics $g_{j}$ on $X$. These 'cylinders' are not metric products. They have the following properties.

5. The projection $\Sigma \times I \rightarrow I$ is a Riemannian submersion over an interval $I=[0,2 j]$, where the interesting behavior is exhibited when $j$ grows without bound.

6. The metric at the endpoints 0 and $2 j$ agrees with that of $\Sigma$.

7. The $(n-3)$-dimensional manifold $K$ has a fixed metric independent of $j$, and is a direct summand in a metric product.

8. For integer values of $x \in I$, each connected component of $C \times T^{1} \times\{x\}$ is isometric to a standard unit square torus.

9. The metric on $C \times T^{1} \times[0,2 j]$ is the 'double' of the metric on $C \times T^{1} \times[0, j]$, in the sense that $C \times T^{1} \times\{x\}$ and $C \times T^{1} \times\{2 j-x\}$ have identical metrics.

Now let $N$ be a non-trivial $(n-1)$-cycle of $X$. By Poincaré duality, the cycle $N$ has non-zero intersection number with one of the connected components, $C_{i}$, of $C$. Hence $N$ induces a non-trivial relative cycle in a neighborhood of $C_{i}$. From now on we will denote this component by $C$.

Note that the volume of $\left(X, g_{j}\right)$ grows linearly in $j$. We will obtain a lower bound for the $(n-1)$-volume of $N$, and therefore for the $(n-1)$-systole of $g_{j}$, which grows faster than the volume of $g_{j}$. Meanwhile, the 1-systole is bounded from below uniformly in $j$. The theorem now follows from the properties of suitable metrics on $T^{2} \times I$ constructed below.

Our technique is calibration by the $(n-1)$-form $\alpha \wedge \mu_{K}=* d z \wedge \mu_{K}$, where $\mu_{K}$ is the volume form of $K$. Here the 2-form $\alpha=* d z$ provides the lower bound for the area of the relative 2-cycle $M$ in Lemma A.3 below. 


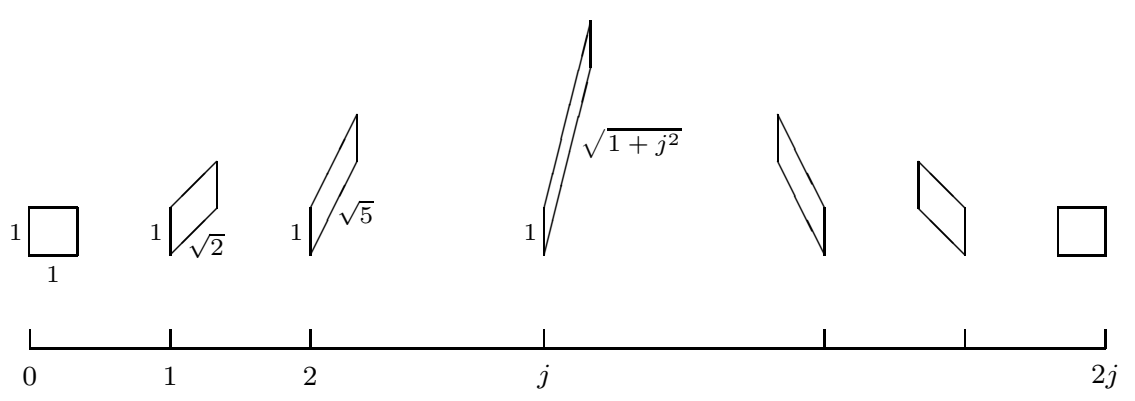

FIgURE 3. Family of parallelograms defining metrics $g_{j}$ on $T^{2} \times I$

The metric on $C \times T^{1} \times[0,2 j]$ is not a direct sum. Consider the subinterval $[0, j] \subset[0,2 j]$. Then $C \times T^{1} \times[0, j]$ can be thought of as a fundamental domain for a $j$-fold cover of a non-trivial torus bundle over the circle, defining either the NIL or SOL geometries (used in [4] and [23], respectively). We will present a description valid for both approaches.

What makes these metrics systolically interesting are the following properties. There is an orientable surface $M^{2} \subset C \times T^{1} \times[0,2 j]$ and a 2-form $\alpha$ on $C \times T^{1} \times[0,2 j]$ such that

(a) $M$ and $C$ have intersection number equal to 1 .

(b) length $\left(M \cap T_{x}^{2}\right) \geq x$ for all $x \in[0, j]$, where $T_{x}^{2}=C \times T^{1} \times\{x\}$.

(c) $\alpha\left(e_{1}, e_{2}\right)=|\alpha(p)|$ for every $p \in M$, where $\left(e_{1}, e_{2}\right)$ is an orthonormal basis of $T_{p} M$ (i.e., the 2-form is maximal in the direction tangent to $M$ ).

(d) The 2-form $\phi(x) \alpha$ is closed, for any function $\phi$.

More specifically, we have the following.

Lemma A.3. Equip the manifold $Y=T^{2} \times I$ with metrics $g_{j}$ defined by

$$
g_{j}(x, y, z)=h(\hat{x})(y, z)+d x^{2},
$$

where $x \in I=[0,2 j], \hat{x}=\min (x, 2 j-x), T^{2}$ is the quotient of the $(y, z)$-plane by the integer lattice, and $h(x)(y, z)=(d z-x d y)^{2}+d y^{2}$ defines a metric on the 2-torus $T^{2} \times\{x\}$ (see Figure [3). Then $Y$ has properties (a)-(d).

Proof. Define the surface $M$ to be the cylinder $T^{1} \times I \subset \mathbb{R}^{2} / \mathbb{Z}$, an open subset of the quotient of the $(y, x)$-plane by unit translation in the $y$-coordinate. The curve $C$ is the projection of the $z$-axis. Set $\alpha=* d z$, the Hodge star of the coordinate 1-form $d z$, for the metric $g_{j}$.

With respect to the metric $g_{j}$, the forms $d x, d y$, and $d z-x d y$ form an orthonormal basis of $T_{(x, y, z)}^{*} Y$. We will need the following calculation of the pointwise norm of the 1-form $d z$ :

$$
|d z|^{2}=|d z-x d y|^{2}+|x d y|^{2}=1+x^{2} .
$$

Now given any surface $M^{\prime} \subset\left(Y, g_{j}\right)$ in the relative homology class of $M$ in $H_{2}(Y, \partial Y)$, we have by Stokes' theorem and properties (d) and (d)

$$
\operatorname{area}\left(M^{\prime}\right) \geq \int_{M^{\prime}} \phi(x) * d z=\int_{M} \phi(x) * d z \geq \int_{1}^{j-1} \sqrt{1+x^{2}} d x \sim j^{2},
$$


for any function $\phi(x)$ with support in $[0, j]$, which is dominated by the function $|d z|^{-1}$ and coincides with it on $[1, j-1]$. This lower bound for the relative 2 -systole is the source of all freedom.

Remark A.4. The metric $g_{j}$ does admit a mod 2 relative 2 -cycle in the same class as $M$, whose area grows linearly in $j$. Namely, consider the 2-chain $a=T^{1} \times[0,2] \subset$ $C \times T^{1} \times[0,2 j]$. Let the 2-chain $b \subset T^{2} \times\{2\}$ be defined by the projection to the torus of the triangle of base 1 (the circle $T^{1}$ ) and altitude 2 (twice the curve $C)$. The boundary of $b$ modulo 2 consists of two circles: image of the base and image of the hypothenuse. Let $c=a+b$. Let $h: T^{2} \times\{0\} \rightarrow T^{2} \times\{1\}$ be the glueing homeomorphism of the NIL bundle which provided the starting point for our construction. Here $h$ is represented by the matrix $\left(\begin{array}{ll}1 & 1 \\ 0 & 1\end{array}\right)$ and is an isometry Let with respect to the metric $g_{j}$.

$$
d=c+h^{2}(c)+h^{4}(c)+h^{6}(c)+\cdots+h^{j-2}(c)
$$

(here we are assuming that $j$ is even). Then $d$ is a mod 2 relative 2-cycle of $T^{2} \times[0, j]$. Doubling it as in item 9 above, we obtain the desired relative cycle, representing the generator of $H_{2}\left(T^{2} \times I, \partial\left(T^{2} \times I\right) ; \mathbb{Z}_{2}\right)$.

For the inequalities involving systoles of complementary dimensions $k$ and $n-k$, the existing results on freedom depend on the divisibility of $k$ by 4 .

Theorem A.5 ([2]). Let $X^{n}$ be a compact, orientable, smooth $n$-dimensional manifold. Assume $X$ is $(k-1)$-connected, where $k<\frac{n}{2}$ and $k$ is not divisible by 4 . Then $X$ is $(k, n-k)$-systolically free.

\section{Appendix B. Freedom PULled BACK}

Our main goal here is to prove Lemma 7.12, which follows from the three lemmas below.

Lemma B.1 (20). Let $Y$ be a triangulable $C W$-complex of dimension n. Let $W=$ $Y \cup_{f} e^{k}$ be a complex obtained from $Y$ by attaching a single cell of dimension $k \leq n-1$, where the attaching map $f$ is triangulable. Let $1 \leq q \leq n-1$. Then the q-systolic freedom of $Y$ implies that of $W$. The same statement is true for $(q, n-q)$-freedom, etc.

Proof. Here the volume of an $n$-dimensional triangulable complex is by definition the sum of volumes of all cells of maximal dimension.

The idea is to insert a long $k$-dimensional cylinder in such a way that a $q$-cycle of volume comparable to the $q$-systole of $Y$ would necessarily have a 'narrow place' somewhere along the cylinder, by virtue of the coarea inequality. We cut the cycle into two pieces at the narrow place, and fill the cut with a small $q$-chain (using the isoperimetric inequality for small $(q-1)$-cycles) to make both pieces into cycles. The lemma now follows from the fact that the cylinder, being of positive codimension, does not contribute to top-dimensional volume.

By the 'long cylinder' metric we mean the following. Let $I=[0, \ell]$, with $\ell \gg 1$ to be determined. Let $S=\operatorname{Im}(f)$ be the image of the attaching map. We may assume that $S$ is a subcomplex of $Y$.

Let $\mathrm{Cyl}_{f}=Y \cup_{f \times\{0\}}\left(S^{k-1} \times I\right)$ be the mapping cylinder of $f$ and $C_{f}=$ $\mathrm{Cyl}_{f} \cup_{\mathrm{id} \times\{\ell\}} D^{k}$ the mapping cone, where $D^{k}$ is a cell of dimension $k \leq n-1$.

Let $g$ be a metric on $Y$. By scaling, we may assume that $\operatorname{sys}_{q}(g) \geq 1$. 
Let $h_{0}$ be the restriction of $g$ to $S \subset Y$. Let $\left(S^{k-1}, h_{1}\right)$ be a round sphere of sufficiently large radius $r \geq 1$ so that the triangulable map $f:\left(S^{k-1}, h_{1}\right) \rightarrow\left(S, h_{0}\right)$ is distance-decreasing, or roughly, $h_{1} \geq h_{0}$. Let $D^{k}$ be a round hemisphere of the same radius as $\left(S^{k-1}, h_{1}\right)$.

We endow the cylinder $S^{k-1} \times I$ with the metric $(1-x) h_{0}+x h_{1}+d x^{2}$ for $0 \leq x \leq 1$ and $h_{1}+d x^{2}$ for $1 \leq x \leq \ell$. We thus obtain a metric on the complex $W=C_{f}$. Denote the resulting metric space by $W(g, \ell)$.

Note that mapping cylinder $\mathrm{Cyl}_{f} \subset W(g, \ell)$ admits a distance-decreasing projection to $\left(Y, g_{j}\right)$ by construction.

Let $p: W(g, \ell) \rightarrow I$ be the map extending to $W$ the projection to the second factor $S^{k-1} \times I \rightarrow I$ on the cylinder, while $p(Y)=0$ and $p\left(D^{k}\right)=\ell$. Let $z$ be a $q$-cycle in $W$. The complex $W$ is not a manifold, but its subspace $S^{k-1} \times I$ is a manifold and we can apply the coarea inequality just in this part of $W$. We obtain the inequality $\operatorname{vol}_{q}(z) \geq \int_{1}^{\ell} \operatorname{vol}_{q-1}\left(z \cap p^{-1}(x)\right) d x$. Hence we can find a regular value $x_{0} \in I$ such that the $(q-1)$-cycle $c=z \cap p^{-1}\left(x_{0}\right)$ satisfies

$$
\operatorname{vol}_{q-1}(c)=\operatorname{vol}_{q-1}\left(z \cap p^{-1}\left(x_{0}\right)\right) \leq \frac{1}{\ell-1} \operatorname{vol}_{q}(z) .
$$

Note that in the case $q=k-1$, the cycle $c$ is empty.

Let us now show that if $Y$ admits a systolically free sequence of metrics $g_{j}$, then so does $W$. (Note that we are not assuming that the inclusion of $Y$ in $C_{f}$ induces a surjective map in $q$-dimensional homology.)

Choose $\ell=\ell(j) \geq \operatorname{sys}_{q}\left(g_{j}\right) \geq 1$. We would like to show that the $q$-volume of non-trivial $q$-cycles in $W\left(g_{j}, \ell\right)$ is comparable to $\operatorname{sys}_{q}\left(g_{j}\right)$. Suppose, on the contrary, that a sequence of cycles $z_{j}$ in $W\left(g_{j}, \ell\right)$ satisfies

$$
\operatorname{vol}_{q}\left(z_{j}\right)=o\left(\operatorname{sys}_{q}\left(g_{j}\right)\right) .
$$

Letting $c_{j}=z_{j} \cap p^{-1}\left(x_{0}\right)$, for suitable $x_{0}$ depending on $j$ as in (2), we obtain $\lim _{j \rightarrow \infty} \operatorname{vol}_{q-1}\left(c_{j}\right)=0$.

We now appeal to the isoperimetric inequality for small cycles (cf. [12], Sublemma 3.4. $B^{\prime}$ ), applied to the cross section $S^{k-1} \times\left\{x_{0}\right\}$ of the cylinder, to obtain a $q$-chain $B_{j}^{q} \subset S^{k-1}$ satisfying

$$
\operatorname{vol}_{q}\left(B_{j}^{q}\right) \leq K \operatorname{vol}_{q-1}\left(c_{j}\right)^{\frac{q}{q-1}} .
$$

In other words, the $(q-1)$-cycle $c_{j}$ can be filled by a chain whose volume also tends to 0 . Clearly, the constant $K$ in the inequality can be chosen independent of the radius $r \geq 1$ of $S^{k-1}$. Let

$$
a_{j}=\left(z_{j} \cap d^{-1}\left(\left[0, x_{0}\right]\right)\right)-B_{j}^{q}
$$

and $b_{j}=z_{j}-a_{j}$. Note that $\left[b_{j}\right]=0$ in $W$ and so $\left[a_{j}\right]=\left[z_{j}\right] \neq 0$. The cycle $a_{j}$ lies in the mapping cylinder which admits a distance-decreasing projection to $\left(Y, g_{j}\right)$.

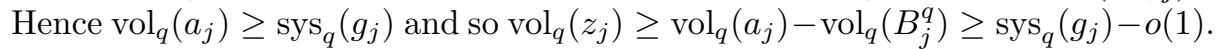
This shows that the systoles of $W$ are not significantly diminished compared to those of $Y$.

Lemma B.2. Let $X$ and $W$ be triangulable $C W$-complexes of dimension $n$. Suppose there is a map $\phi: X \rightarrow W$ inducing a monomorphism on $H_{q}$. Then the $q$-systolic freedom of $W$ implies that of $X$. The same statement holds for $(q, n-q)$ freedom, etc. 
Proof. Choose a simplicial structure on $W$. By the cellular approximation theorem, a continuous map from $X$ to $W$ is homotopic to a simplicial map. As in [1], we can replace it by a map which has the following property with respect to suitable triangulations of $X$ and $W$ : on each simplex of $X$, it is either a diffeomorphism onto its image or the collapse onto a wall of positive codimension. Let $p$ be the maximal number of $n$-simplices of $X$ mapping diffeomorphically to an $n$-simplex of $W$. Since cells of dimension $\leq(n-1)$ do not contribute to $n$-dimensional volume, the pullback of the metric on $W$ is a positive quadratic form on $X$ whose $n$-volume is at most $p$ times that of $W$. This form is piecewise smooth and satisfies natural compatibility conditions along the common face of each pair of simplices.

Lemma B.3. If a smooth compact $n$-manifold $X$ admits systolically free piecewise smooth metrics, then it also admits systolically free smooth metrics.

Proof. To construct a smooth metric from a piecewise smooth one, we proceed as in [1]. Given a piecewise smooth metric $g$, compatible along the common face of each pair of adjacent simplices, we choose a smooth metric $h$ on $X$ such that $h>g$ at every point (in the sense of lengths of all tangent vectors). Let $N$ be a regular neighborhood of small volume of the $(n-1)$-skeleton of the triangulation. Choose an open cover of $X$ consisting of $N$ and the interiors $U_{i}$ of all $n$-simplices. Using a partition of unity subordinate to this cover, we patch together the metrics $\left.g\right|_{U_{i}}$ and $\left.h\right|_{N}$. The new metric dominates $g$ for each tangent vector to $M$. In particular, the volume of a cycle is not decreased. Meanwhile, $n$-dimensional volume is increased no more than the volume of the regular neighborhood.

The piecewise smooth metric on $X$ may a priori not be compatible with its smooth structure, since the triangulation may not be smooth. To clarify this point, denote the triangulation by $s$, and the piecewise smooth metric by $g$. Consider a smooth triangulation $s^{\prime}$, and approximate the identity map of $X$ by a map $\phi$, simplicial with respect to $s^{\prime}$ and $s$. The pullback metric $g^{\prime}=\phi^{*}(g)$ is then adapted to the smooth triangulation $s^{\prime}$, so we may apply to it the argument with the regular neighborhood $N$.

We have thus obtained a smooth positive form on $X$. We make it definite without significantly increasing its volume by adding a small multiple of a positive definite form. The lower bounds for the $q$-systole are immediate from the injectivity of the map $X \rightarrow W$ on $H_{q}$.

We now establish the systolic freedom of a model space by a 'long cylinder' argument.

Lemma B.4. Let $X$ and $Y$ be q-systolically free $n$-complexes, and let $S$ be an $(n-2)$-complex which is simultaneously a subcomplex of $X^{(n-2)}$ and $Y^{(n-2)}$. Let $I$ be an interval. Then the complex

$$
W=X \cup(S \times I) \cup Y
$$

is also q-systolically free.

Proof. Let $\epsilon>0$, and let $(X, g)$ and $(Y, h)$ be metrics satisfying

$$
\frac{\operatorname{vol}(g)^{\frac{q}{n}}}{\operatorname{sys}_{q}(g)}<\epsilon \quad \text { and } \quad \frac{\operatorname{vol}(h)^{\frac{q}{n}}}{\operatorname{sys}_{q}(h)}<\epsilon .
$$

We will construct a metric on $M$ satisfying a similar inequality for $2 \epsilon$. For this purpose, assume that $g$ and $h$ are scaled to the same value of the $q$-systole. 
Let $\mu$ be a metric on $S$ dominating both (the pullbacks of) $g$ and $h$. Let $I=[0, \ell]$. We define a metric on $W$ to be equal to $\mu \oplus d t^{2}$, when $t \in[1, \ell-1] \subset I$. Near the extremities of $I$, we patch the metric to make a continuous transition to $g$ and $h$, as in the proof of Lemma B.1. The systolic freedom of $W$ follows by applying the coarea inequality as before. Namely, a non-trivial $q$-cycle in $W$ can be cut at a narrow place and decomposed, by filling the cut, into two pieces, one of which admits a distance-decreasing projection to $(X, g)$, and the other to $(Y, h)$. One of the pieces must be non-trivial (since they add up to a non-trivial cycle), hence bounded below by $\operatorname{sys}_{q}(X)=\operatorname{sys}_{q}(Y)$. The contribution from the filling cycle is negligible by the isoperimetric inequality for small cycles.

\section{Appendix C. A Mayer-Vietoris argument}

Lemma C.1. Let $X$ be a closed, orientable, smooth 4-manifold. Let $C \subset X$ be a smoothly embedded closed curve in $X$, representing an element in the torsion-free part of $H_{1}(X)$. Let $Y$ be the result of surgery along $C$. Let $Z$ be the cobordism determined by the surgery, and let $i: X \rightarrow Z$ and $j: Y \rightarrow Z$ be the inclusions. There is then an isomorphism $\psi: H_{2}(X) \rightarrow H_{2}(Y)$ such that $j_{*} \circ \psi=i_{*}$.

Proof. Let $k: S^{1} \rightarrow X$ be an embedding with $k\left(S^{1}\right)=C$. By assumption, $k_{*}: H_{1}\left(S^{1}\right) \rightarrow H_{1}(X)$ is a monomorphism. Let $X_{-}$be a neighborhood of $S^{1}$ (homeomorphic to $S^{1} \times D^{3}$ ), and $X_{+}$the closure of its complement. Let $i^{ \pm}: X_{ \pm} \rightarrow X$ and $k^{ \pm}: S^{1} \times S^{2} \rightarrow X_{ \pm}$be the inclusions. Consider the following fragment of the Mayer-Vietoris sequence:

$$
\begin{array}{r}
H_{3}(X) \stackrel{\stackrel{\partial}{\rightarrow}}{\rightarrow} H_{2}\left(S^{1} \times S^{2}\right) \rightarrow H_{2}\left(X_{-}\right) \oplus H_{2}\left(X_{+}\right) \stackrel{\left(i_{*}^{-}, i_{*}^{+}\right)}{\longrightarrow} H_{2}(X) \rightarrow \\
H_{1}\left(S^{1} \times S^{2}\right) \stackrel{k_{*}^{-}+k_{*}^{+}}{\longrightarrow} H_{1}\left(X_{-}\right) \oplus H_{1}\left(X_{+}\right) \stackrel{\left(i_{*}^{-}, i_{*}^{+}\right)}{\longrightarrow} H_{1}(X) \rightarrow 0 .
\end{array}
$$

We have: $H_{2}\left(X_{-}\right)=0$, the map $\partial$ is surjective (under Poincaré duality and universal coefficients, it is the dual of $k_{*}: H_{1}\left(S^{1}\right) \rightarrow H_{1}(X)$, which is injective), and $k_{*}^{-}+k_{*}^{+}$is injective, with image $H_{1}\left(X_{-}\right)$. Thus, the maps $i_{*}^{+}: H_{1}\left(X_{+}\right) \rightarrow H_{1}(X)$ and $i_{*}^{+}: H_{2}\left(X_{+}\right) \rightarrow H_{2}(X)$ are isomorphisms.

The surgery replacing $X_{-}$by $D^{2} \times S^{2}$ yields $Y=D^{2} \times S^{2} \cup X_{+}$. Let $j^{-}$: $D^{2} \times S^{2} \rightarrow Y, j^{+}: X_{+} \rightarrow Y$, and $\ell: S^{1} \times S^{2} \rightarrow D^{2} \times S^{2}$ be the inclusions. The corresponding Mayer-Vietoris sequence is

$$
\begin{aligned}
& H_{2}\left(S^{1} \times S^{2}\right) \stackrel{\ell_{*}+k_{*}^{+}}{\longrightarrow} H_{2}\left(D^{2} \times S^{2}\right) \oplus H_{2}\left(X_{+}\right) \stackrel{\left(j_{*}^{-}, j_{*}^{+}\right)}{\longrightarrow} H_{2}(Y) \rightarrow \\
& H_{1}\left(S^{1} \times S^{2}\right) \stackrel{\ell_{*}+k_{*}^{+}}{\longrightarrow} H_{1}\left(D^{2} \times S^{2}\right) \oplus H_{1}\left(X_{+}\right) .
\end{aligned}
$$

We have: $H_{1}\left(D^{2} \times S^{2}\right)=0$, the map $\ell_{*}: H_{2}\left(S^{1} \times S^{2}\right) \rightarrow H_{2}\left(D^{2} \times S^{2}\right)$ is an isomorphism, and $k_{*}^{+}: H_{1}\left(S^{1} \times S^{2}\right) \rightarrow H_{1}\left(X_{+}\right)$is injective (since $k_{*}$ is injective, $i_{*}^{+}$is an isomorphism, and $\left.i_{*}^{+} \circ k_{*}^{+}=k_{*}\right)$. Thus, $j_{*}^{+}: H_{2}\left(X_{+}\right) \rightarrow H_{2}(Y)$ is an isomorphism.

The desired isomorphism is obtained by combining the two isomorphisms above:

$$
\psi=j_{*}^{+} \circ\left(i_{*}^{+}\right)^{-1}: H_{2}(Y) \rightarrow H_{2}(X)
$$

It remains to verify that $j_{*} \circ \psi=i_{*}$. Recall that, by definition, $Z=X \times I \cup h^{2}$, where the handle $h^{2}=D^{2} \times D^{3}$ is attached along $X_{-}=S^{1} \times D^{3}$. We thus have a diffeomorphism $Z \cong X_{+} \times I \cup\left(X_{-} \times I \cup h^{2}\right)$. Under this decomposition, the inclusions $i \circ i^{+}$and $j \circ j^{+}$of $X_{+}$into the boundary components of $Z$ correspond to the inclusions $\iota_{0}$ and $\iota_{1}$ of $X_{+}$into the boundary components of $X_{+} \times I$. The 
identity map $X_{+} \times I \rightarrow X_{+} \times I$ is a homotopy $\iota_{0} \simeq \iota_{1}$. This implies $i \circ i^{+} \simeq j \circ j^{+}$, hence $i_{*} \circ i_{*}^{+}=j_{*} \circ j_{*}^{+}$, and the conclusion follows.

\section{Appendix D. Spinning}

The process of spinning was introduced in knot theory by E. Artin, and was extended to closed manifolds by S. Cappell [7].

Let $M^{n}$ be a compact, simply-connected, smooth manifold of dimension $n \geq 3$. The result of performing surgery on $S^{1} \times M$ along $S^{1} \times *$ is called the spin of $M$. There is a choice of framing involved in the surgery, corresponding to $\pi_{1}(\mathrm{SO}(n))=$ $\mathbb{Z}_{2}$. The spin obtained by surgery with trivial framing is denoted by $\sigma(M)$; the 'twisted' spin, by $\sigma^{\prime}(M)$.

Note that $\sigma(M)=\partial\left(D^{2} \times M_{\circ}\right)$, where $M_{\circ}=M^{n} \backslash \operatorname{int} D^{n}$ is a punctured copy of $M$. Indeed, $\sigma(M)=S^{1} \times M^{n} \backslash\left(S^{1} \times \operatorname{int} D^{n}\right) \cup D^{2} \times S^{n-1}=S^{1} \times M_{\circ} \cup D^{2} \times S^{n-1}=$ $\partial D^{2} \times M_{\circ} \cup D^{2} \times \partial M_{\circ}=\partial\left(D^{2} \times M_{\circ}\right)$.

If $M$ admits an effective circle action with non-empty fixed point set, then it is easily shown that $\sigma^{\prime}(M) \cong \sigma(M)$. In general, though, the two spins of $M$ are not even homotopy equivalent.

Lemma D.1 (25]). Both spins of $S^{1} \times S^{n-1}$ can be identified with the connected sum $S^{1} \times S^{n} \# S^{n-1} \times S^{2}$.

Proof. We have $\sigma\left(S^{1} \times S^{n-1}\right)=\partial W$, where $W=D^{2} \times\left(S^{1} \times S^{n-1}\right)$ 。. The manifold $W$ admits a handle decomposition $W=D^{n+2} \cup h^{1} \cup h^{n-1}$, where the attaching spheres form a trivial link $S^{0} \cup S^{n-2} \subset S^{n+1}$. Thus, $W$ can be identified with the boundary connected sum $\left(D^{n+2} \cup h^{1}\right) \sharp\left(D^{n+2} \cup h^{n-1}\right) \cong S^{1} \times D^{n+1} \sharp S^{n-1} \times D^{3}$. Passing to boundaries, we obtain

$$
\sigma\left(S^{1} \times S^{n-1}\right) \cong S^{1} \times S^{n} \# S^{n-1} \times S^{2} .
$$

Rotation in the second factor of $S^{1} \times S^{n-1}$ provides a circle action with nonempty fixed point set. Thus, $\sigma^{\prime}\left(S^{1} \times S^{n-1}\right) \cong \sigma\left(S^{1} \times S^{n-1}\right)$.

Corollary D.2. The manifold $S^{2} \times S^{n-1}$ is obtained from $S^{1} \times S^{1} \times S^{n-1}$ by performing surgery along the curves $\gamma_{1}=S^{1} \times * \times *$ and $\gamma_{2}=* \times S^{1} \times *$.

Proof. First consider the case where both surgeries are done with trivial framing. Surgery along $\gamma_{1}$ yields the spin $\sigma\left(S^{1} \times S^{n-1}\right)$. Under the diffeomorphism in (3), the image of the curve $\gamma_{2}$ on the left side corresponds to the curve $S^{1} \times *$ in the factor $S^{1} \times S^{n}$ on the right side. Surgery on $S^{1} \times S^{n}$ along $S^{1} \times *$ yields $\sigma\left(S^{n}\right)=S^{n+1}$, and the corollary follows.

The other cases are similar. It suffices to note that $S^{n}$ admits an effective circle action with fixed points, and so $\sigma^{\prime}\left(S^{n}\right) \cong \sigma\left(S^{n}\right)$.

\section{Appendix E. Whitehead products and Hilton's theorem}

The product of two spheres decomposes as $S_{a}^{i} \times S_{b}^{j}=\left(S_{a}^{i} \vee S_{b}^{j}\right) \cup_{f} e^{i+j}$, where $f: S^{i+j-1} \rightarrow S^{i} \vee S^{j}$ is the attaching map of the top cell, so that $[f]=0$ in $\pi_{i+j-1}\left(S^{i} \times S^{j}\right)$. This decomposition defines a $\mathbb{Z}$-bilinear pairing

$$
[,]: \pi_{i}(X) \times \pi_{j}(X) \rightarrow \pi_{i+j-1}(X)
$$

on the homotopy groups of an arbitrary space $X$. Here the element $[a, b]$ in the case of our bouquet is defined by the map $f$. More precisely, if $\alpha \in \pi_{i}(X)$ and 
$\beta \in \pi_{j}(X)$, then $[\alpha, \beta]$ is the obstruction to extending the map $a \vee b: S^{i} \vee S^{j} \rightarrow X$ to the whole of $S^{i} \times S^{j}$, where $a$ and $b$ are arbitrary representatives of $\alpha$ and $\beta$.

The above operation, which generalizes the usual commutator map in $\pi_{1}$, is called the Whitehead product. It is natural with respect to continuous maps, and satisfies identities analogous to the Lie bracket. See [28] for more details.

Theorem E.1 (Hilton [18]). The first unstable homotopy group of a bouquet of spheres is given by:

$$
\pi_{2 m-1}\left(\vee S_{r}^{m}\right)=\oplus_{r} \pi_{2 m-1}\left(S_{r}^{m}\right) \oplus\left(\oplus_{r<s} \mathbb{Z}\left[e_{r}, e_{s}\right]\right) .
$$

Meanwhile, $\pi_{j}\left(\vee S_{r}^{m}\right)=\oplus_{r} \pi_{j}\left(S_{r}^{m}\right)$ for $m \leq j \leq 2 m-2$.

For example, $\pi_{3}\left(S^{2} \vee S^{2}\right)=\mathbb{Z} \oplus \mathbb{Z} \oplus \mathbb{Z}$. The first $\mathbb{Z}$-summand corresponds to $\pi_{3}\left(S^{2}\right)$, and is generated by the Hopf map $h: S^{3} \rightarrow S^{2}$. This generator is written unambiguously (by virtue of the absence of torsion in this dimension) as $h=\frac{1}{2}[a, a]$, where $a$ is the fundamental class of $S^{2}$. Similarly, the second $\mathbb{Z}$ summand is generated by $\frac{1}{2}[b, b]$. The last $\mathbb{Z}$-summand is generated by $[a, b]$.

\section{REFERENCES}

[1] I. Babenko, Asymptotic invariants of smooth manifolds, Russian Acad. Sci. Izv. Math. 41 (1993), 1-38.

[2] I. Babenko and M. Katz, Systolic freedom of orientable manifolds, Ann. Sci. École Norm. Sup. 31 (1998); available at math.DG/9707102.

[3] I. Babenko, M. Katz and A. Suciu, Volumes, middle-dimensional systoles, and Whitehead products, Math. Res. Lett. 5 (1998), 461-471.

[4] L. Bérard Bergery and M. Katz, Intersystolic inequalities in dimension 3, Geom. Funct. Anal. 4 (1994), 621-632.

[5] M. Berger, Du côté de chez Pu, Ann. Sci. École Norm. Sup. 5 (1972), 1-44.

[6] Systoles et applications selon Gromov, Séminaire N. Bourbaki, exposé 771, Astérisque 216 (1993), 279-310.

[7] S. Cappell, Superspinning and knot complements, In: Topology of Manifolds (Proc. Inst. Univ. of Georgia, Athens, Ga., 1969), Markham, Chicago, IL, 1970, pp. 358-383.

[8] B. Eckmann, Über die Homotopiegruppen von Gruppenräumen, Comment. Math. Helv. 14 (1942), 234-256.

[9] H. Federer, Real flat chains, cochains and variational problems, Indiana Univ. Math. J. 24 (1974), 351-407.

[10] M. Freedman, Personal communication, June 23, 1998.

[11] H. Gluck, D. Mackenzie, and F. Morgan, Volume-minimizing cycles in Grassmann manifolds, Duke Math. J. 79 (1995), 335-404.

[12] M. Gromov, Filling Riemannian manifolds, J. Differential Geom. 18 (1983), 1-147.

[13] _ Pseudoholomorphic curves in symplectic manifolds, Invent. Math. 82 (1985), 307347.

[14] — Systoles and intersystolic inequalities, In: Actes de la Table Ronde de Géométrie Différentielle (Luminy, 1992), Sémin. Congr., vol. 1, Soc. Math. France, Paris, 1996, pp. 291362.

[15] _ Metric structures for Riemannian and non-Riemannian spaces, Progr. Math., vol. 152, Birkhäuser, Boston, MA, 1998.

[16] _ Personal communication, August 7, 1998.

[17] M. Gromov, J. Lafontaine, P. Pansu, Structures Métriques pour les Variétés Riemanniennes, Cedic, 1981.

[18] P. J. Hilton, On the homotopy groups of the union of spheres, J. London Math. Soc. 30 (1955), 154-172.

[19] M. Katz, Counterexamples to isosystolic inequalities, Geom. Dedicata 57 (1995), 195-206.

[20] Systolically free manifolds, Appendix D in 15 .

[21] A. Lundell and S. Weingram, The topology of $C W$ complexes, University Series in Higher Math., Van Nostrand Reinhold, New York, 1969. 
[22] J. Milnor, Lectures on the h-cobordism theorem, Princeton Univ. Press, Princeton, NJ, 1965.

[23] C. Pittet, Systoles on $S^{1} \times S^{n}$, Differential Geom. Appl. 7 (1997), 139-142.

[24] J.-P. Serre, Groupes d'homotopie et classes de groupes abeliens, Ann. of Math. 58 (1953), $258-294$.

[25] A. Suciu, Iterated spinning and homology spheres, Trans. Amer. Math. Soc. 321 (1990), $145-157$.

[26] D. Sullivan, Genetics of homotopy theory and the Adams conjecture, Ann. of Math. 100 (1974), 1-79.

[27] G. W. Whitehead, A generalization of the Hopf invariant, Ann. of Math. 51 (1950), 192-237.

[28] Elements of homotopy theory, Grad. Texts in Math., vol. 61, Springer-Verlag, New York, 1978.

UMR 9973, Département de Mathématiques, Université de Nancy 1, B.P. 239, 54506 VAndoeuvre, France

Current address: School of Mathematical Sciences, Sackler Faculty, Tel Aviv University, Ramat Aviv, Tel Aviv 69978 Israel

E-mail address: katzm@math.tau.ac.il

Department of Mathematics, Northeastern University, Boston, MA 02115, USA

E-mail address: alexsuciu@neu.edu 